\title{
The Role of Multidetector Row Computed Tomography in Biliary Tract Malignancy
}

\author{
Indira Narayanaswamy*, Akshaya Reddy Erasu, Ram Prakash HV \\ Department of Radio-diagnosis, Vydehi Institiute of Medical Sciences and Research Centre, EPIP Area, Bangalore, Karnataka, India
}

Email address:

drindiraniranjan@gmail.com (I. Narayanaswamy),drakshayreddy@gmail.com (A. R. Erasu)

\section{To cite this article:}

Indira Narayanaswamy, Akshaya Reddy Erasu, Ram Prakash HV. The Role of Multidetector Row Computed Tomography in Biliary Tract Malignancy. Cancer Research Journal. Vol. 3, No. 5, 2015, pp. 104-109. doi: 10.11648/j.crj.20150305.13

\begin{abstract}
Early diagnosis of biliary tract tumors is important in accurate staging, to choose the best possible treatment and improve their prognosis. Multidetector row Computed Tomography is an ideal method to evaluate patients with suspected biliary tract obstruction and thereby biliary tract malignancy. Aims and Objective: To evaluate the accuracy of Multidetector row Computed Tomography (MDCT) in evaluation of level of obstruction and to study the imaging features of various causes of biliary tract malignancy. Material and Method: MDCT of 40 patients with clinically suspicion of biliary tract obstruction were prospectively reviewed and only malignant causes were included in the study. Final diagnosis was based on endoscopic retrograde cholangiopancreatography, surgical and histopathological diagnosis. The MDCT diagnosis and final diagnosis was compared for level and imaging features of malignant lesions. Results: The sensitivity, specificity, PPV, NPV and diagnostic accuracy of MDCT in determining the level of malignant obstruction is $100 \%, 83.33 \%, 97.77 \%, 100 \%, 98 \%$, and cause of malignant obstruction is $97.73 \%, 83.33 \%, 97.72 \%, 83.33 \%, 96 \%$. MDCT with its high sensitivity and specificity values plays an essential role in the diagnosis of malignant tumors of biliary tract and for the evaluation of therapeutic options. Conclusion: MDCT has a vital role to play in biliary tract malignancy with its high degree of accuracy in establishing the level of obstruction and in defining the imaging features of the malignant lesions.
\end{abstract}

Keywords: Biliary Tract Obstruction, Multidetector Row Computed Tomography, Benign, Malignant

\section{Introduction}

Early diagnosis of biliary tract tumors is important in accurate staging, to choose the best possible treatment and improve their prognosis. The role of a radiologist therefore is important in early diagnosis and in accurately delineating the level and the cause of obstruction, thus helping in staging as well as preoperative assessment of tumor respectability. ${ }^{1}$

Ultrasound is a non-invasive and cost effective imaging technique available for evaluating biliary tract obstruction. Ultrasound demonstrates the presence of biliary tract obstruction by identifying dilated bile ducts but has a sensitivity of 55\%-95\% and specificity of $71 \%-96 \%{ }^{2}$

The role of magnetic resonance cholangiography (MRCP) is well established in this field as the most reliable noninvasive technique. ${ }^{3}$ However, some drawbacks, such as contraindication in patients with pacemakers and ferromagnetic implants, for claustrophobic patients, long examination times exceeding 30 minutes and limited availability of scanners still limit its use. MRCP is expensive and has limitations such as interference from intraluminal gas, pneumobilia and flow artifacts ${ }^{4}$.

The search for noninvasive diagnostic tools is an emerging medical need, in order to avoid invasive, costly and physician intensive procedures such as endoscopic retrograde cholangiopancreatography (ERCP). ${ }^{5}$

In the past, axial conventional $\mathrm{CT}$ could not provide adequate information of biliary abnormalities since the total depiction of these ducts was not suitable for evaluation in axial planes. ${ }^{5}$ MDCT with its ability to obtain volume dataset with sub-millimeter spatial resolution allows the optimal display of bile duct by using multiplanar reconstruction (MPR) and minimal intensity projection (MinIP) without compromising on image quality. ${ }^{6}$ The optimal contrast enhancement of both hepatic and pancreatic parenchyma afforded by the spiral technique allows excellent visualization of the biliary system from the 
level of intrahepatic ducts to the ampulla of Vater. ${ }^{7}$ In addition, spiral $\mathrm{CT}$ enables a high rate of detection of hepatic metastases, which when present support a neoplastic etiology for biliary tract obstruction. ${ }^{7}$

The purpose of the present study is to evaluate the accuracy of MDCT in evaluation of level and imaging features of various causes of biliary tract malignancy by comparing with ERCP, surgical or histopathological findings.

\section{Material and Methods}

A prospective study was conducted in Department of Radio-diagnosis, Vydehi Institute of Medical Sciences and Research Centre, Bangalore on 60 patients with clinical suspicion of jaundice or ultrasound detected biliary tract dilataion between Dec 2012 to Nov 2014. Only proved cases of malignancies were included. Patients with benign causes, who are less than 10 years, pregnant ladies, patients allergic to contrast and patients with renal insufficiency were excluded.

Informed consent was taken from all the patients and the study was approval by Ethics committee.

\subsection{Examination Technique}

All MDCT examinations was performed on empty stomach after giving $800 \mathrm{ml}$ of oral Mannitol, with a 16channel MDCT scanner (GE Light Speed RT). CT was performed in unenhanced, arterial, portal and venous phases. Unenhanced CT with $1 \mathrm{~mm}$ collimation of the upper abdomen was performed after which, contrast enhanced CT images was obtained using a $18 \mathrm{sec}$ delay during hepatic arterial dominant phase and a 45 to 50 seconds delay during portal dominant phase and 90 seconds delay during venous dominant phase after the initiation of IV injection of $80 \mathrm{ml}$ non-ionic contrast material (IOHEXOL) at the rate of $3.5 \mathrm{ml} / \mathrm{s}$ using a power injector.

\subsection{Image Interpretation}

Interpretation of the CT images will be performed on a workstation equipped with a software tool, which allows generation of maximum intensity projections (MIPs), Volume Renderings (VRs), and Multiplanar Reconstructions (MPRs). The reconstructed images were evaluated for the level and imaging features of obstruction.

All the cases were comprehensively evaluated and correlated with relevant history, clinical, ERCP, intraoperative and histopathological findings.

\subsection{Statistical Methods}

Data obtained was tabulated using version 22 of the Statistical Package for Social Sciences (SPSS, published
SPSS Inc.) and subjected to appropriate statistical analysis. Open EPI software was used to calculate sensitivity, specificity, positive predictive value, negative predictive value and diagnostic accuracy of MDCT for detection of the level of biliary tract obstruction and the cause of biliary tract malignancy were evaluated.

\section{Results}

Out of 40 patients, $26(65 \%)$ were males with mean age of 50.1 years and $14(35 \%)$ were fenales with mean age of 45.5 years. there was male predominance with male to female ratio being 1.8:1.

Table 1. Nature Of Obstruction.

\begin{tabular}{lll}
\hline & Frequency & Percent \\
\hline Benign & 20 & 33.3 \\
Malignant & 40 & 66.7 \\
Total & 60 & 100 \\
\hline
\end{tabular}

The incidence of malignant cause of biliary tract obstruction was $66.7 \%$ as compared to $33.3 \%$ of benign casue of biliary tract obstruction. The ratio of malignant cause to benign cause is $2: 1$. The mean age of patients with benign cause was 42.7 years and that with malignant cause was 50.6 years.

Out of $40(60 \%)$ cases characterized as malignant cause of biliary tract obstruction, 1 case $(2 \%)$ diagnosed on MDCT as extrahepatic cholangiocarcinoma was proven to be carcinoma head of pancreas on final diagnosis by intraoperative and histopathalogical examination.

Table 2. Malignant Cause of Obstruction Cross tabulation.

\begin{tabular}{lll}
\hline & On CT & Final Diagnosis \\
\hline Cholangiocarcinoma & 16 & 15 \\
Carcinoma Head of Pancreas & 6 & 6 \\
Periampullary Carcinoma & 4 & 5 \\
Carcinoma Gall Bladder & 3 & 3 \\
Hepatocellular Carcinoma & 2 & 2 \\
Duodenal Malignancy & 1 & 1 \\
Lymph nodes & 8 & 8 \\
Total & 40 & 40 \\
\hline
\end{tabular}

Among malignant causes, cholangiocarcinoma presented in $48 \%$ (16 patients), carcinoma head of pancreas presented in $18 \%$ (6 patients), periampullary carcinoma presented in $12 \%$ (4 patients), carcinoma gall bladder presented in $9 \%(3$ patients), hepatocellular carcinoma presented in $6 \%$ (2 patients) and duodenal malignancy presented in $3 \%$ (1 patients) and lymph nodes $20 \%$ (8 patients). 1 case diagnosed on MDCT as extrahepatic cholangiocarcinoma turned out to be carcinoma head of pancreas on final diagnosis. 


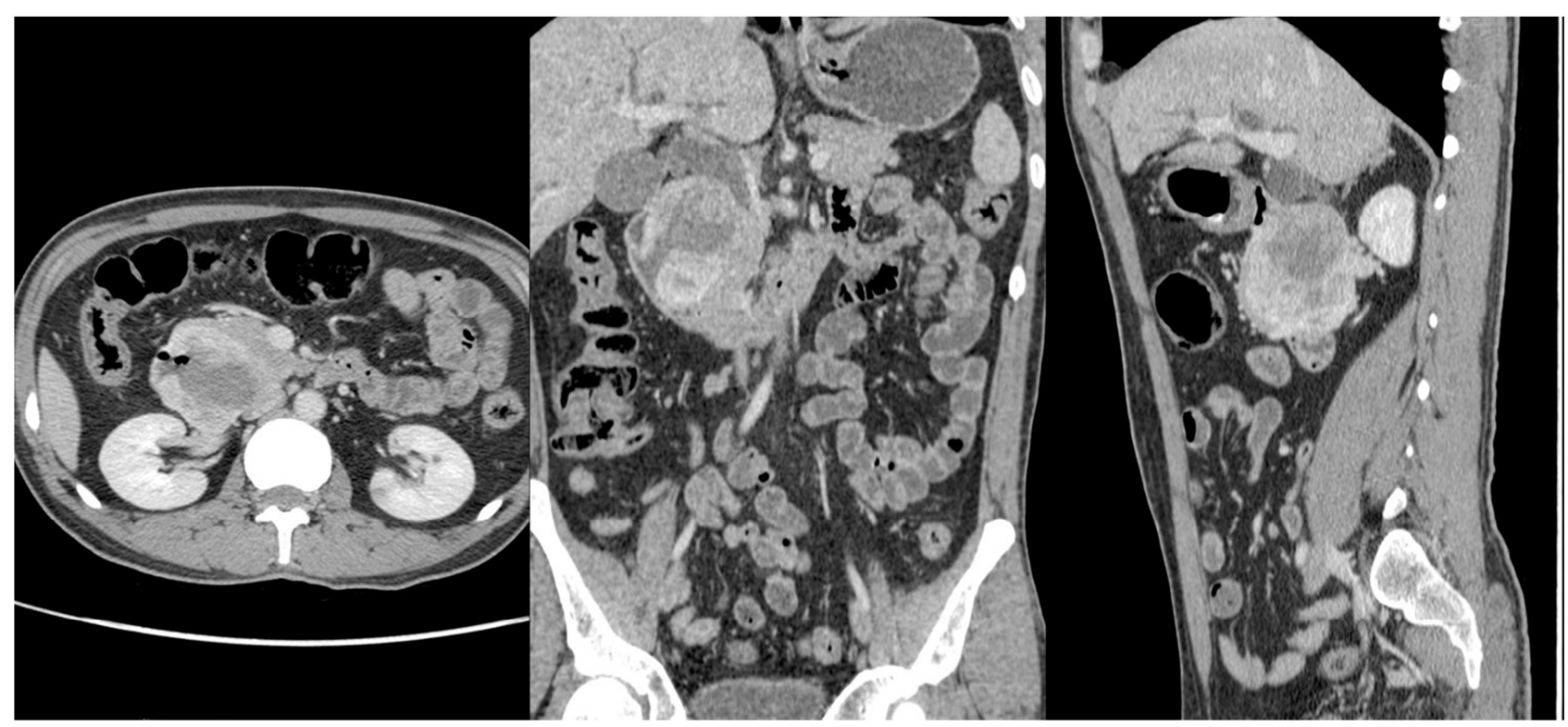

Figure 1. Multiplanar images of periampullary carcinoma.

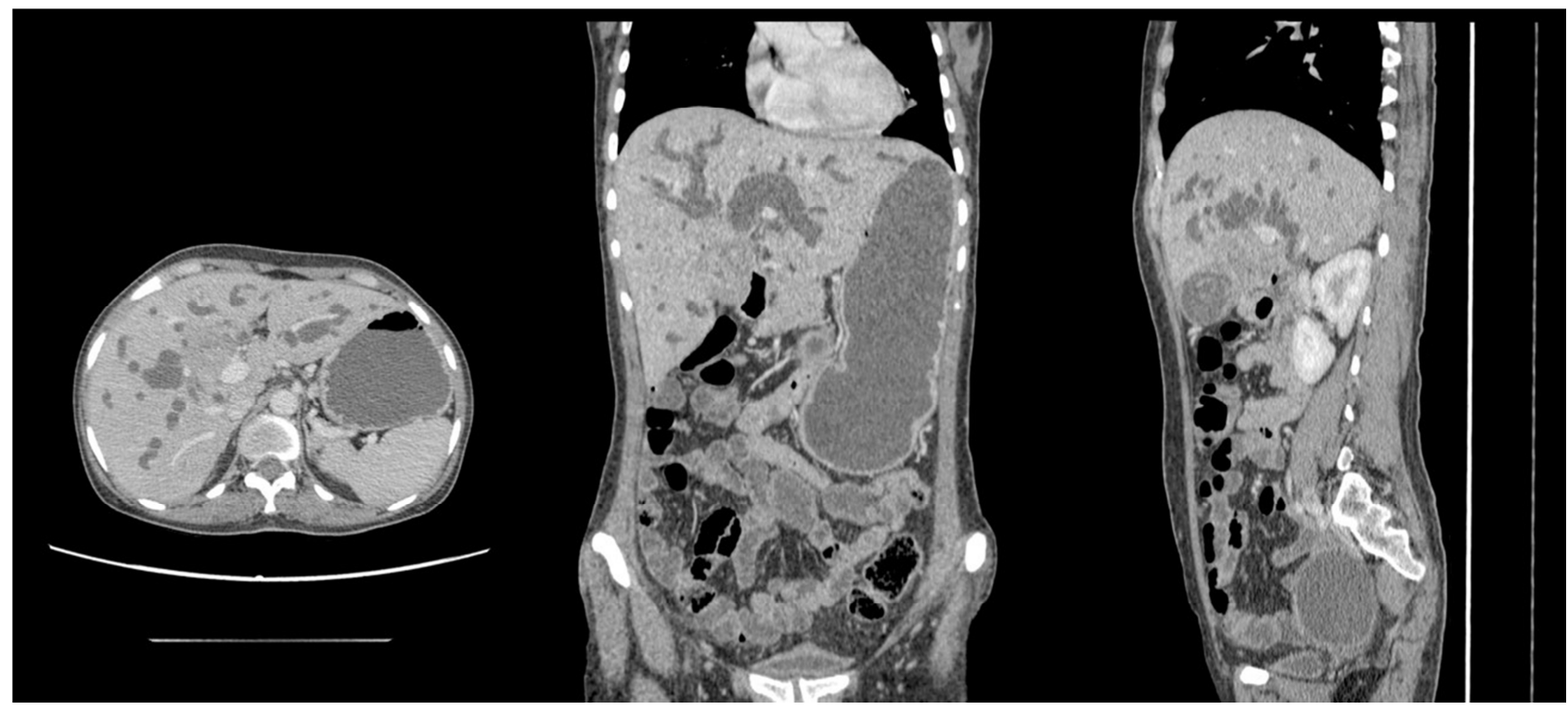

Figure 2. Multiplanar images of klatskin's tumour.

Table 3. Cross tabulation Of Diagnosis on Computed Tomography For Cause and Level Of Malignant lesions of Biliary Tract Obstruction.

\begin{tabular}{llllll}
\hline \multirow{2}{*}{ Diagnosis on Computed Tomography } & \multicolumn{2}{l}{ Level of Obstruction } & & Supra Pancreatic & Intra Pancreatic \\
\cline { 2 - 5 } & Intra Hepatic & Porto Hepatic & 4 & 2 & $16(40 \%)$ \\
\hline Cholangiocarcinoma & 5 & 5 & 0 & 6 & $6(15 \%)$ \\
Carcinoma Head Of Pancreas & 0 & 0 & 0 & 4 & $4(10 \%)$ \\
Periampullary Carcinoma & 0 & 0 & 0 & 1 & $3(7.5 \%)$ \\
Carcinoma Gall Bladder & 0 & 2 & 0 & 0 & $2(5 \%)$ \\
Hepatocellular Carcinoma & 1 & 1 & 0 & 1 & $1(2.5 \%)$ \\
Duodenal Malignancy & 0 & 0 & 4 & 0 & $8(20 \%)$ \\
Lymph nodes & 0 & 4 & $20(16 \%)$ & $14(54 \%)$ & $40(100 \%)$ \\
Total & $6(15 \%)$ & $12(30 \%)$ & & & \\
\hline
\end{tabular}

Table 4. Statistical Analysis of MDCT for the Evaluation of Biliary Tract Obstruction.

\begin{tabular}{llllll}
\hline & Sensitivity & Specificity & PPV & NPV & Accuracy \\
\hline Level of malignant obstruction & $100.00 \%$ & $83.33 \%$ & $97.77 \%$ & $100.00 \%$ & $98 \%$ \\
Cause of malignant obstruction & $97.73 \%$ & $83.33 \%$ & $97.72 \%$ & $83.33 \%$ & $96 \%$ \\
\hline
\end{tabular}




\section{Discussion}

Multidetector row Computed Tomography remains the most commonly used technique for imaging patients with suspected abdominal abnormalities. ${ }^{1}$ It allows the rapid acquisition of multiphase data sets and continuous raw data for detailed reconstruction into three-dimensional (3D) images. ${ }^{1}$

Invasive methods such as endoscopic retrograde cholangiopancreatography and percutaneous transhepatic cholangiography were used earlier for the evaluation of the biliary tract. ${ }^{7}$ The optimal contrast enhancement of both hepatic and pancreatic parenchyma afforded by the spiral technique allows excellent visualization of the biliary system from the level of intrahepatic ducts to the ampulla of Vater. ${ }^{7}$ In addition, spiral CT enables a high rate of detection of hepatic metastases, which when present support a neoplastic etiology for biliary tract obstruction. ${ }^{7}$ This study has been conducted to assess the role of MDCT in the detection of malignant causes of biliary tract obstruction, discuss their level of obstruction and study their imaging features. The findings of MDCT were further compared with Endoscopic Retrograde Cholangiopancreatography, surgical diagnosis and histopathalogical diagnosis.

In the present study, MDCT demonstrated evidence of biliary tract dilatation in 40 patients with peak incidence of biliary tract obstruction in the age group belonging to 30 to 60 years of age with a mean age of 48 years. The youngest patient of our study was 30 years old and the oldest was 75 years. 26 cases $(52 \%)$ are males with a mean age of 50.1 years and 24 cases $(48 \%)$ are females with a mean age of 45.5 years.

This study revealed higher incidence at $66.6 \%(40 / 60)$ of malignant causes, as opposed to $33.3 \%$ (20/40) incidence of benign cause of obstructive jaundice. The mean age of patients with benign cause was 42.7 years and that with malignant cause was 50.6 years. Our study correlates with the findings of studies by Huang JQ et al (1993) ${ }^{8}$, Sharma MP et al (1999) ${ }^{9}$, Martin DF et al (2001) ${ }^{10}$ and Siddque K et al (2008) ${ }^{11}$ seeking to evaluate the etiological spectrum of obstructive jaundice.

MDCT accurately characterized 40 patients $(66.6 \%)$ as having malignant cause of biliary tract obstruction, among which cholangiocarcinoma presented in $40 \%$ (16 patients), carcinoma head of pancreas presented in $15 \%$ (6 patients), periampullary carcinoma presented in 10\% (4 patients), carcinoma gall bladder presented in 7.5 (3 patients), hepatocellular carcinoma presented in 5\% (2 patients) and duodenal malignancy presented in $2.5 \%$ ( 1 patient). 1 case diagnosed on MDCT as extrahepatic cholangiocarcinoma turned out to be carcinoma head of pancreas on final diagnosis.

A study by Ferrari FS et al $(2005)^{6}$ quotes Helical CT has an accuracy of $93 \%$ for differentiating benign from malignant biliary obstruction. Findings in a study by O'Connor KW et al $(1983)^{12}$ and Lerttumnongtum $\mathrm{P}$ et al (2002), ${ }^{13}$ the corresponding sensitivity of CT was $66.7 \%-90.9 \%$, specificity $77.8 \%$ - $100.0 \%$, PPV $89.3 \%-100.0 \%$, NPV
$87.5 \%-94.4 \%$, and $\mathrm{p}$ value was $<0.001$. In the present study, the statistical analysis of MDCT in determining the malignant cause of obstruction had sensitivity, specificity, specificity, PPV and Diagnostic Accuracy was 96.97\%, 98\%, 100\% and $95 \%$ respectively with $\mathrm{p}$ value of $<0.001 \%$ which is statistically significant.

Obstructing lesions of biliary tract such as neoplasms and lymph nodes and stricture secondary to primary or lymph nodes were apparent on MDCT in 40 cases, with final/primary diagnoses correctly suggesting the CT findings in 39 cases (97.5\%). One case diagnosed on MDCT as extrahepatic cholangiocarcinoma turned out to be carcinoma head of pancreas on final diagnosis Findings of focal, asymmetrical ductal wall thickening of $6 \mathrm{~mm}$ led to an erroneous presumptive diagnosis of cholangiocarcinoma. Few limitations of the $\mathrm{CT}$ are radiation exposure and use of intravenous contrast and hence cannot be used in patients suffering from renal failure.

Levels of obstruction and imagimg features of obstructing lesions:

\subsection{Pancreatic Level Including Periampullary Region}

There were 16 cases $(40 \%)$ of obstruction at the intrapancreatic level including the ampullary region among which, 6 cases $(37.5 \%)$ were due to carcinoma head of pancreatic of which 3 cases showed dilatation of the main pancreatic duct, 4 cases $(25 \%)$ were due to periampullary carcinoma which was seen as polypoid mass at region of ampulla with abrupt dilatation of common duct or double duct sign where both common bile duct and pancreatic duct is dilated. Two cases $(12.5 \%)$ with cholangiocarcinoma, which was seen with delayed and sustained enhancement of contrast and one case $(6.25 \%)$ of carcinoma gall bladder infiltrating the ampullary region. Duodenal malignancy was seen in one case as thickening and enhancement of the duodenal wall. CT also detected hepatic metastases in four patients with pancreatic head masses. Regional lymphadenopathy involving the peripancreatic, paraaortic, or celiac nodal groups was noted in three patients. Three patients manifested vascular encasement of the superior mesenteric artery and/or vein by a pancreatic mass.

\subsection{Suprapancreatic Level}

Eight $(20 \%)$ patients had obstruction at the supra-pancreatic level. In four $(50 \%)$ patients cholangiocarcinoma was the diagnosis on basis of focal, asymmetrical thickening of the common bile duct wall; ERCP confirmed the diagnosis. Lymph nodes were seen in $4(50 \%)$ cases as low attenuation masses at the coeliac and superior mesenteric levels.

\subsection{Level of Porta Hepatis}

Twelve patients $(30 \%)$ had obstruction at the porto-hepatic level. Six patients $(50 \%)$ manifested with low-attenuation mass lesions, with five $(83.3 \%)$ due to cholangiocarcinoma, which was seen with delayed and sustained enhancement of 
contrast and one (16.7\%) was hepatocellular carcinoma which showed neovascularity and early wash out of contrast. Two patients $(16.6 \%)$ presented with infiltration of the confluence of right and left hepatic ducts by an adjacent gallbladder carcinoma, which revealed narrowing of the proximal common bile duct for a length of $8 \mathrm{~mm} .4$ patients (33.3\%) had metastatic lymph nodes seen as low attenuation masses at the porta hepatis.

\subsection{Intrahepatic Level}

Six patients $(15 \%)$ had intrahepatic biliary obstruction who presented with low-attenuation mass lesions, with five $(83.33 \%)$ due to cholangiocarcinoma, which was seen with delayed and sustained enhancement of contrast and one $(16.66 \%)$ ccase of hepatocellular carcinoma showed neovascularity and early wash out. MDCT proved to be highly sensitive $(100 \%)$ in detecting all cases of intrahepatic and proximal common hepatic duct obstruction due to infiltrating neoplasms. These lesions typically manifest by irregular regions of low attenuation surrounding the intrahepatic ducts, and are clearly accentuated by the excellent enhancement of surrounding hepatic parenchyma. ${ }^{14}$

The pancreas in $62 \%$ of the patients was normal, $30 \%$ had mass lesion (including malignant infiltration) of which $12 \%$ is diagnosed as carcinoma head of pancreas. Main Pancreatic Duct was dilated in $26 \%$ of patients and normal in rest of the $74 \%$. Although the normal pancreas showed only minimal enhancement with routine dynamic CT, the optimal contrast enhancement achieved with the spiral technique accentuates the difference between normal pancreatic parenchyma and the typically hypodense pancreatic cancer that lead to biliary obstruction. In addition to adequate vascular enhancement, thin sections through the pancreas are also critical for the accurate detection of small neoplasms. ${ }^{14}$ Previous studies showed a significant incidence of small pancreatic cancers manifest on $\mathrm{CT}$ by abrupt termination of the common duct without a mass visualized. ${ }^{15}$ MDCT improves direct visualization of these small cancers. Furthermore, MDCT provides superior opacification of the peripancreatic vasculature and thus clearly depicts vascular encasement or invasion. An accurate assessment of vascular invasion is of critical importance in determining potential resectability. ${ }^{15}$

When obstruction is at the level of the distal common bile duct, the differential diagnosis must include, in addition to pancreatic adenocarcinoma, ampullary carcinoma, primary cholangiocarcinoma of the distal duct. ${ }^{16}$

In our study, significant associated findings such vascular complications, ascites, hepatic metastasis, pancreatic metastasis, skeletal metastasis and malignant lymphadenopathy were detected in majority of the patients, where ERCP did not provide equivalent findings.

In a study by Midwinter MJ et al (1999), ${ }^{17}$ on MDCT, the specificity and confidence with which a diagnosis can be suggested are further increased by the high rate of detection of such associated findings as hepatic metastases, adenopathy in the porta hepatis or peripancreatic nodal groups.

\section{Conclusions}

MDCT clearly has achieved a vital role in the evaluation of the biliary tract malignancy. Spiral technique augments the diagnostic capability of CT, primarily due to excellent parenchymal enhancement of both the liver and the pancreas and to the volume dataset with lack of spatial misregistration. Biliary tract obstruction can be clearly localized to levels such as the distal intrahepatic ducts, porta hepatic, mid-distal common bile duct (suprapancreatic), pancreatic head or ampullary. The imaging features of most malignant lesions are so specific that confident diagnosis can be made out.

\section{References}

[1] A. Joshi, K. Rajpal, K. Kakadiya, A. Bansal. Role of CT and MRCP in Evaluation of biliary tract obstrucyion. Curr Radiol Rep (2014) 2: 722.

[2] Tasneem Lalani, Corey AC, Max PR, Mark EB, et al: ACR appropriateness criteria on jaundice. American College of Radiology 2012.

[3] Guibaud L, Bret PM, Reinhold C, Mostafa A, Barkun ANG Diagnosis of choledocholithiasis. Value of MR cholangiography. Am J Roentgenol (1994) 163: 847-850.

[4] David V, Reinhold C, Hochman M, Chuttani R, McKee J, Waxman I, et al. Pitfalls in the interpretation of MR cholangiopancreatography. AJR Am J Roentgenol. 1998; 170: 1055-9.

[5] Moore KL and TVN Persaud: The Developing Human Clinically Oriented Embryology, 7th ed. Elsevier Science (WB Saunders), Philadelphia (2003), p. 78.

[6] Ferrari FS, Fantozzi F, Tasciotti L, Vigni F, Scotto F, Frasci P. US, MRCP, CCT and ERCP: a comparative study in 131 patients with suspected biliary obstruction. Med Sci Monit. 2005; 11:MT8-18.

[7] Bluemke DA, Fishman EK. Spiral CT of the liver. AJR 1993; 160: 787-792.

[8] Huang JQ, Bao XJ, Lu XH. The common causes and differential diagnosis of malignant jaundice. Zhonghua Nei Ke Za Zhi 1993; 32: 400-4.

[9] Sharma MP, Ahuja V. Aetiological spectrum of obstructive jaundice and diagnostic ability of ultrasonography: a clinician's perspective. Trop Gastroenterol 1999; 20: 167-9.

[10]Martin DF, Laasch HU. The biliary tract. In: Grainger RG, Allison D eds. Grainger \& Allison's Diagnostic Radiology-A textbook of medical imaging, 4th ed. Churchill Livingstone, Harcourt publishers limited, London: 2001.

[11] Siddigue K, Ali Q, Mirza S, Jamil A, Ehsan A, Latif S, et al. Evauation of the aetiological spectrum of obstructive jaundice. J Ayub Med Coll Abbotabad 2008; 20: 62-6.

[12] O'Connor KW. A blinded prospective study comparing four current noninvasive approaches in the differential diagnosis of medical versus surgical jaundice. Gastroenterology 1983; 84:1498-504. 
[13] Lerttumnongtum P, Muttarak M, Wasanavijit K. Clinics in Diagnostic Imaging. Singapore Medical Journal 2002; 43:592-3.

[14] Susan HW, Elliot KF. Biliary tract obstruction the role of Spiral CT in detection and definition of disease. Clinical Imaging 1997; 21: 27-34.

[15] Megibow AJ. Pancreatic adenocarcinoma: designing the examination to evaluate the clinical questions. Radiology 1992; 183: 297-303.
[16] Schulte SJ, Baron RL, Teefey SA, et al. CT of the extrahepatic bile ducts: wall thickness and contrast enhancement in normal and abnormal ducts. AJR 190; 154:79-85.

[17] Midwinter MJ, Beveridge CJ, Wilsdon JB, Bennett MK, Baudouin CJ, Charnley RM: Correlation between spiral computed tomography, endoscopic ultrasonography and findings at operation in pancreatic and ampullary tumors. Br J Surg 1999, 86: 189-193. 